\title{
Performance Analysis of AODV, DSDV and I-DSDV Routing Protocols in MANET
}

\author{
Mr. S. Parthiban ${ }^{1}$, Mrs. T. Priyadarsini ${ }^{2}$ \\ Assistant Professor, Dept of ECE, V.R.S. College of Engineering \& Technology, Arasur, Villupuram, Tamil Nadu ${ }^{1}$ \\ ISTE Member $^{1}$ \\ Assistant Professor, Dept of ECE, V.R.S. College of Engineering \& Technology, Arasur, Villupuram, Tamil Nadu ${ }^{2}$ \\ ISTE Member, IAENG Member, IRED Associate Member ${ }^{2}$
}

\begin{abstract}
Ad hoc networks are characterized by a lack of infrastructure, and by a random and quickly changing network topology; thus the need for a robust dynamic routing protocol that can accommodate such an environment. To improve the packet delivery ratio of Destination-Sequenced Distance Vector (DSDV) routing protocol in mobile ad hoc networks with high mobility, a message exchange scheme for its invalid route reconstruction is being used. Three protocols AODV, DSDV and I-DSDV were simulated using NS-2 package and were compared in terms of packet delivery ratio, end to end delay and routing overhead in different environment; varying number of nodes, speed and pause time. Simulation results show that I-DSDV compared with DSDV, it reduces the number of dropped data packets with little increased overhead at higher rates of node mobility but still can't compete with AODV in higher node speed and number of node.
\end{abstract}

Keywords: DSDV, link breakage, message scheme, ad hoc network.

\section{INTRODUCTION}

A mobile ad-hoc network (MANET) is a network composed of mobile nodes mainly characterized by the absence of any centralized coordination or fixed infrastructure, which makes any node in the network act as a potential router. MANETs are also characterized by a dynamic, random and rapidly changing topology. This makes the classical routing algorithms fail to perform correctly, since they are not robust enough to accommodate such a changing environment. Consequently, more and more research is being conducted to find optimal routing algorithms that would be able to accommodate for such networks.

In MANETs, communication between mobile nodes always requires routing over multi-hop paths. Since no infrastructure exists and node mobility may cause frequent link failure, it is a great challenge to design an effective and adaptive routing protocol. Many restrictions should be well considered, such as limited power and bandwidth.

Destination-Sequenced Distance Vector routing protocol (DSDV) [1] is a typical routing protocol for MANETs, which is based on the Distributed Bellman-Ford algorithm. In DSDV, each route is tagged with a sequence number which is originated by the destination, indicating how old the route is. Each node manages its own sequence number by assigning it two greater than the old one (call an even sequence number) every time. When a route update with a higher sequence number is received, the old route is replaced. In case of different routes with the same sequence number, the route with better metric is used.
Updates are transmitted periodically or immediately when any significant topology change is detected. There are two ways of performing routing update: "full dump", in which a node transmits the complete routing table, and "incremental update", in which a node sends only those entries that have changed since last update. To avoid fluctuations in route updates, DSDV employs a "settling time" data, which is used to predict the time when route becomes stable. In DSDV, broken link may be detected by the layer-2 protocol [2], or it may instead be inferred if no broadcasts have been received for a while from a former neighbouring node.

In this paper the performance comparison between three routing protocols, namely AODV (Adhoc On Demand Distance Vector), DSDV (Destination Sequenced Distance Vector) and the Improvement of DSDV (I-DSDV). While all routing protocols use sequence numbers to prevent routing loops and to ensure the freshness of routing information, AODV and DSDV differ drastically in the fact that they belong to two different routing families [3]. Namely, AODV is a reactive protocol (routes are only generated on demand, in order to reduce routing loads), and DSDV is a proactive protocol (with frequent updates of routing tables regardless of need). The rest of this paper is organized as follows. Section 2 reports a review of previous related works especially for network selection algorithms. In section 3, we present architecture of proposed system, include NFDM in sketch and detail of implemented system. In section 4 the result of experiments has been described. Finally paper concludes in section 5 . 


\section{RELATED WORK}

The Ad hoc On Demand Distance Vector (AODV) routing algorithm is a routing protocol designed for adhoc mobile networks [6] [7]. AODV is capable of both unicast and multicast routing [8]. It is an on demand algorithm, meaning that it builds routes between nodes only as desired by source nodes. It maintains these routes as long as they are needed by the sources. Additionally, AODV forms trees which connect multicast group members. The trees are composed of the group members and the nodes needed to connect the members. AODV uses sequence numbers to ensure the freshness of routes. It is loop-free, self-starting, and scales to large numbers of mobile nodes [8].

AODV builds routes using a route request / route reply query cycle. When a source node desires a route to a destination for which it does not already have a route, it broadcasts a route request (RREQ) packet across the network. Nodes receiving this packet update their information for the source node and set up backwards pointers to the source node in the route tables. In addition to the source node's IP address, current sequence number, and broadcast ID, the RREQ also contains the most recent sequence number for the destination of which the source node is aware. A node receiving the RREQ may send a route reply (RREP) if it is either the destination or if it has a route to the destination with corresponding sequence number greater than or equal to that contained in the RREQ. If this is the case, it unicasts a RREP back to the source. Otherwise, it rebroadcasts the RREQ. Nodes keep track of the RREQ's source IP address and broadcast ID [8]. If they receive a RREQ which they have already processed, they discard the RREQ and do not forward it.

As the RREP propagates back to the source, nodes set up forward pointers to the destination. Once the source node receives the RREP, it may begin to forward data packets to the destination. If the source later receives a RREP containing a greater sequence number or contains the same sequence number with a smaller hop count, it may update its routing information for that destination and begin using the better route.

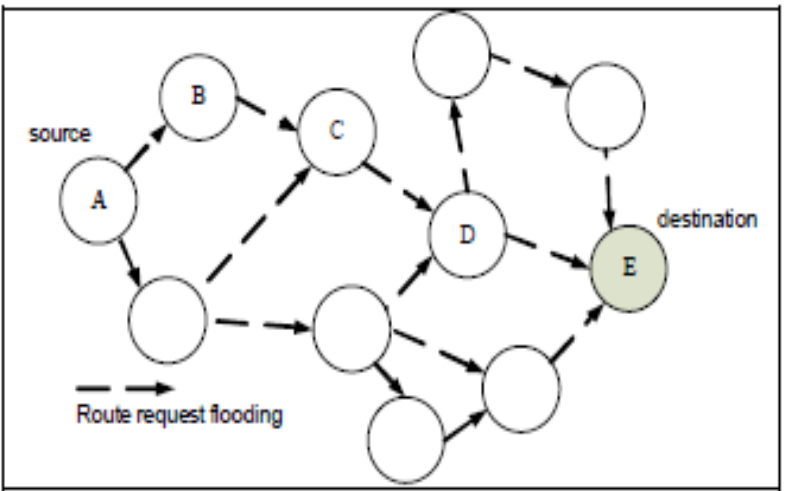

Figure 1: Route Request (RREQ) flooding

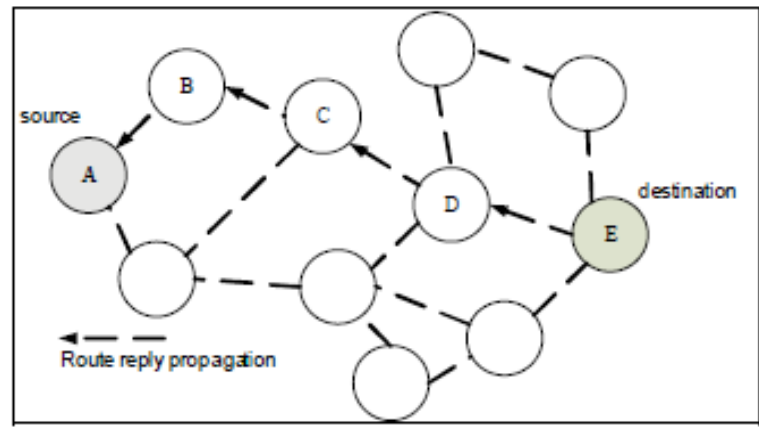

Figure 2: Route Reply (RREP) propagation

As long as the route remains active, it will continue to be maintained. A route is considered active as long as there are data packets periodically travelling from the source to the destination along that path. Once the source stops sending data packets, the links will time out and eventually be deleted from the intermediate node routing tables. If a link break occurs while the route is active, the node upstream of the break propagates a route error (RERR) message to the source node to inform it of the now unreachable destination(s). After receiving the RERR, if the source node still desires the route, it can reinitiate route discovery.

Destination-Sequenced Distance-Vector Routing (DSDV) [14] is a table-driven routing scheme for ad hoc mobile networks based on the Bellman-Ford algorithm. It was developed by C. Perkins and P.Bhagwat in 1994 [1]. The main contribution of the algorithm was to solve the Routing Loop problem. Each entry in the routing table contains a sequence number, the sequence numbers are generally even if a link is present; else, an odd number is used. The number is generated by the destination, and the emitter needs to send out the next update with this number [1] [10]. Routing information is distributed between nodes by sending full dumps infrequently and smaller incremental updates more frequently.

DSDV was one of the early algorithms available. It is quite suitable for creating ad hoc networks with small number of nodes [1]. Since no formal specification of this algorithm is present there is no commercial implementation of this algorithm. Many improved forms of this algorithm have been suggested.

DSDV requires a regular update of its routing tables, which uses up battery power and a small amount of bandwidth even when the network is idle. Whenever the topology of the network changes, a new sequence number is necessary before the network reconverges; thus, DSDV is not suitable for highly dynamic networks. (As in all distance-vector protocols, this does not perturb traffic in regions of the network that are not concerned by the topology change.)

\section{IMPROVEMENT OF DSDV}

In DSDV the low packet delivery is due to the fact that, it uses stale routes in case of broken links [2] [9]. In DSDV the existence of stale route does not imply that there is no 
Vol. 5, Issue 12, December 2016

valid route to the destination. The packets can be forwarded thru other neighbors who may have routes to the destination. When an immediate link from the host says ' $A$ ' to the destination say ' $T$ ' breaks, the proposed protocol creates a temporary link thru a neighbor which has a valid route to the desired destination. The temporary link is created by sending one-hop ROUTE-REQUEST and ROUTE-ACK messages. The host ' $A$ ' upon finding the next hop broken link broadcasts a one-hop ROUTEREQUEST packet to all its neighbors.

In turn, the neighbors returns the ROUTE-ACK if it has a valid route to the destination and the host ' $A$ ' is not the next hop on the route from the neighbor to the destination. Each entry in the routing table has an additional entry for route update time. This update time is embedded in the ROUTE-ACK packet and is used in selecting a temporary route. In case of receiving multiple ROUTE-ACK with the same number of minimum hops, ad hoc host ' $\mathrm{S}$ ' chooses that route which has the latest update time.

Figure 3 shows how host ' $A$ ' creates a provisional route to the destination ' $T$ ', when the intermediate link from ' $A$ ' to 'B' breaks. Host ' $A$ ' suspends sending packets (Figure 3(a)). After which it broadcasts ROUTE-REQUEST packets to its immediate one hop neighbors. The Ad Hoc hosts $\mathrm{C}, \mathrm{E}$, and $\mathrm{G}$ responds with ROUTE ACK packets along with hop count and the route update time to Ad Hoc host A (Figure 3 (b)). Table 1 shows the snapshot of the routing information received by Ad Hoc host A. From the table it can be seen that, Ad Hoc Host $\mathrm{C}$ and $\mathrm{E}$ have the same value for hop count metric, but the routing update time for $\mathrm{E}$ is greater than that of $\mathrm{C}$, meaning the path thru $\mathrm{E}$ is updated more recently. Therefore Host A resumes sending packets to the destination $\mathrm{T}$ (Figure 3(c)). Later on whenever any Ad Hoc host moves in the range of the host A then the routing table of host A gets updated by the regular DSDV routing process. Then the updated route will be taken for forwarding the packets from the host A to the destination $\mathrm{T}$.

The whole process is shown as in Figure 3

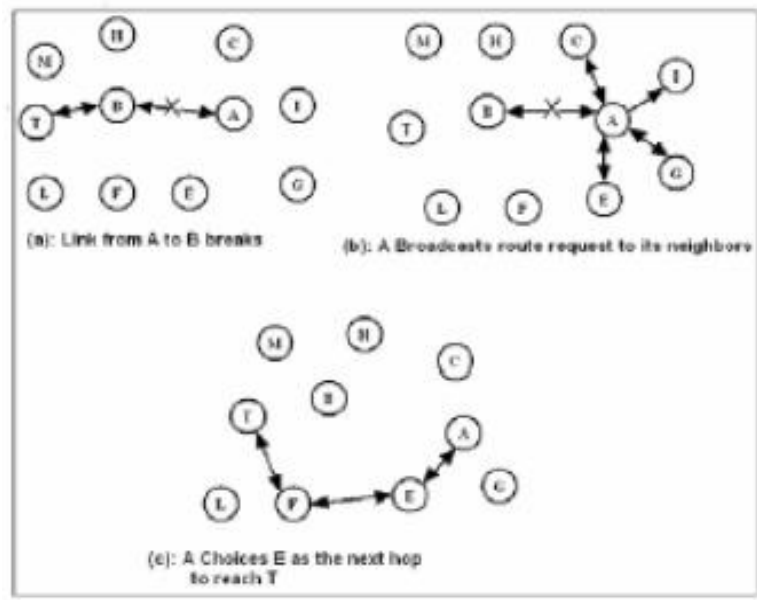

Figure 3: Creation of provisional route in Node " $A$ "
Table 1: Route Update at Host A

\begin{tabular}{|c|c|c|c|}
\hline Neighbor & No,of Hops & Vin Yode & Ipdate Time \\
\hline C & 2 & $\mathrm{H}$ & 1765 \\
\hline$E$ & 2 & $\mathrm{~F}$ & 1860 \\
\hline G & 3 & E & 1050 \\
\hline I & 3 & A & 805 \\
\hline
\end{tabular}

\section{EXPERIMENTAL RESULTS}

The simulation is conducted in three different scenarios. In the first scenario, the comparison of the three routing protocols is compared in various numbers of nodes. The number of nodes is set to $5,10,15,20,25,30$ and 35 nodes.

In the second scenario, the routing protocols are evaluated in different pause time while the number of nodes and the node speed are fixed. The node speed is set to $20 \mathrm{~m} / \mathrm{s}$ and the number of nodes is set to 20 nodes. The pause time are set to $0,50,100,150,200,250,300,350$ and 400 second. In the third scenario, the routing protocols are evaluated in different node speed. The number of nodes is fixed to 30 nodes.

\subsection{Various Numbers of Nodes}

In this scenario, all the three routing protocol are evaluated based on the three performance metric which are Packet Delivery Fraction, End-to-End Delay and the Routing Overhead. The simulation environments for this scenario are:-

- Various number of node which are 5, 10, 15, 20, 25, 30

and 35 nodes

- Packet size is set to 1400 Bytes

- Area size is set to $1000 \times 1000$ flat areas

- Node Speed is fixed to $20 \mathrm{~m} / \mathrm{s}$

- Random Way Point mobility model is used

\subsubsection{Packet Delivery Fraction}

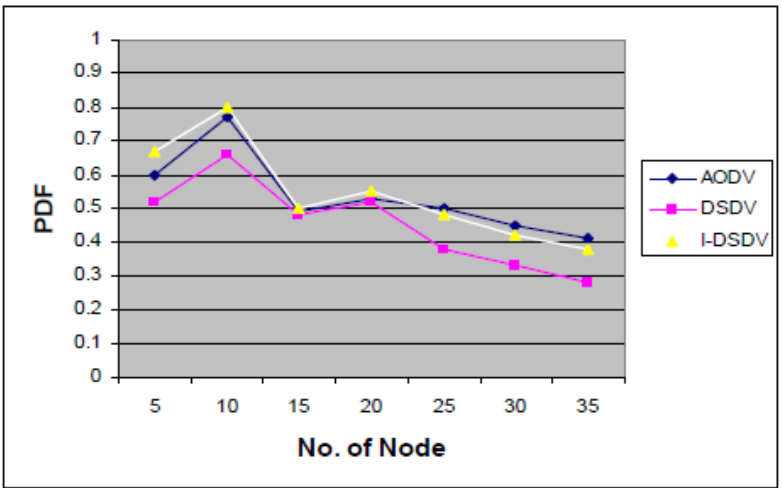

Figure 4: Packet Delivery Fraction in Scenario 1

Based on the Figure 4, it is shown than AODV perform better when the number of nodes increases because nodes become more stationary will lead to more stable path from 
Vol. 5, Issue 12, December 2016

source to destination DSDV performance dropped as - Various pause times which are 0, 50, 100, 150, 200, 250, number of nodes increase because more packets dropped 300,350 , and 400

due to link breaks. I-DSDV is better than DSDV - Simulation time $=400 \mathrm{~s}$

especially when the number of nodes is between 20 and $\bullet$ Number of nodes is fixed to 20 nodes

35. I-DSDV improved the PDF since it find new route to - CBR Packet size is set to 1400 Bytes

destination when link breaks existed.

\subsubsection{End-to-End Delay}

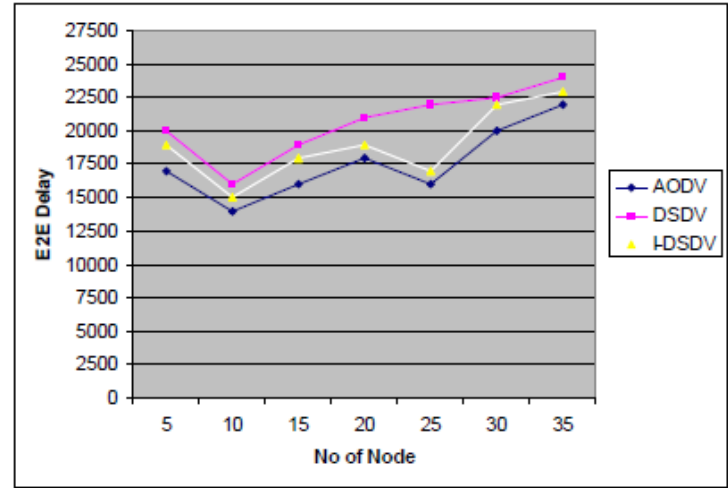

Figure 5: End to End Delay in Scenario 1

AODV didn't produce so much delay even the number of nodes increased. It is better the other two protocols. The performance of I-DSDV is slight better than DSDV especially when the number of nodes between 15 and 30 . It shows that, the I-DSDV protocol improved the DSDV but slightly lower than AODV when the nodes is higher.

\subsubsection{Routing Overhead}

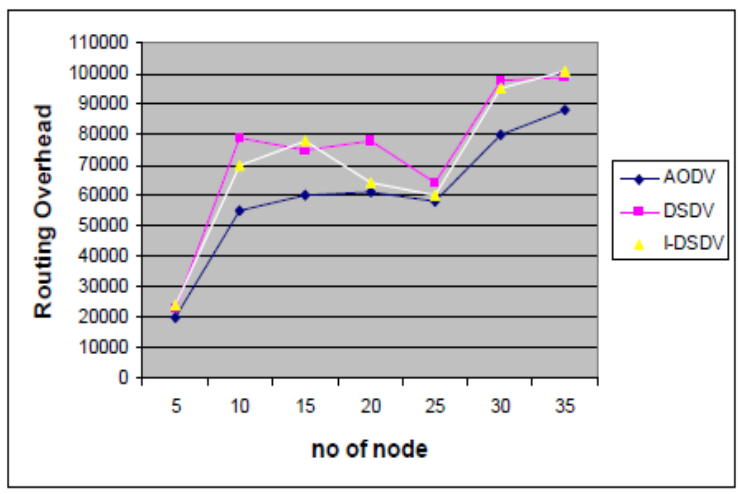

Figure 6: Routing Overhead in Scenario 1

From Figure 6, DSDV less prone to route stability compared to AODV. For AODV, the routing overhead is not as affected as generated in DSDV. I-DSDV generates less routing overhead compared to DSDV. I-DSDV didn't effected by the number nodes.

\subsection{Various Pause Time}

In this scenario, all the three routing protocol are evaluated based on the three performance metric which are Packet Delivery Fraction, End-to-End Delay and the Routing Overhead. The simulation environments for this scenario are:-
- Area size is set to $1000 \times 1000$ flat areas

- Node Speed is fixed to $20 \mathrm{~m} / \mathrm{s}$

- Random Way Point mobility model is used

\subsubsection{Packet Delivery Fraction}

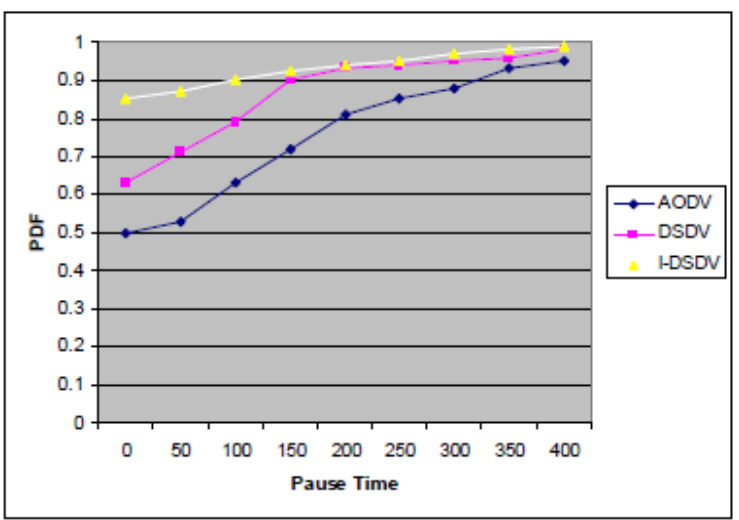

Figure 7: Packet Delivery Fraction in Scenario 2

Based on Figure 7, contrast to DSDV, I-DSDV performs better. It delivers more than $85 \%$ data packet regardless to mobility rate. For DSDV, it performs poorly as the pause time decreased which drop to less than $70 \%$ when pause time is 0. For AODV, it shows significant dependence on route stability, thus its PDF is lower when the pause time decreased

\subsubsection{End-to-End Delay}

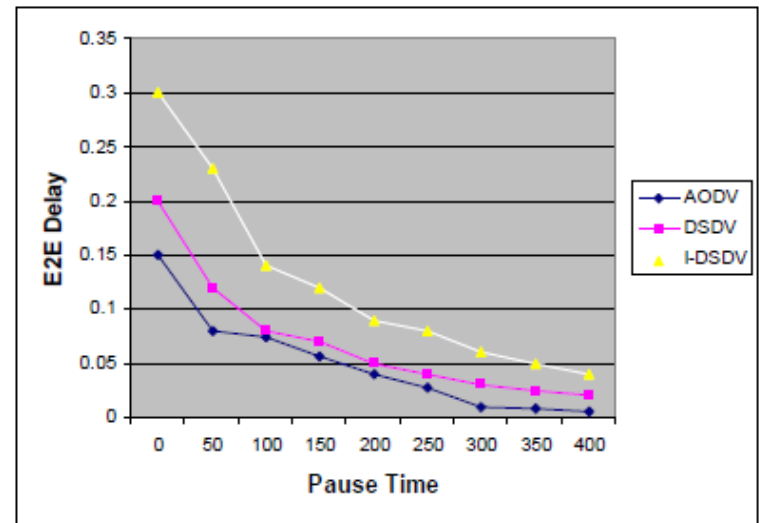

Figure 8: End to End Delay in Scenario 2

Based on Figure 8, I-DSDV exhibits longer average endto-end delay all the time regardless to node mobility rate compared to the other two protocols. I-DSDV uses a message exchange scheme to create a loop-free route to destination when link breaks, and then buffered data packets are transmitted through new route. So it needs longer end-to-end delay time compare to others. AODV 
Vol. 5, Issue 12, December 2016

exhibits shortest end-to-end delay of data packet compare to others.

\subsubsection{Routing Overhead}

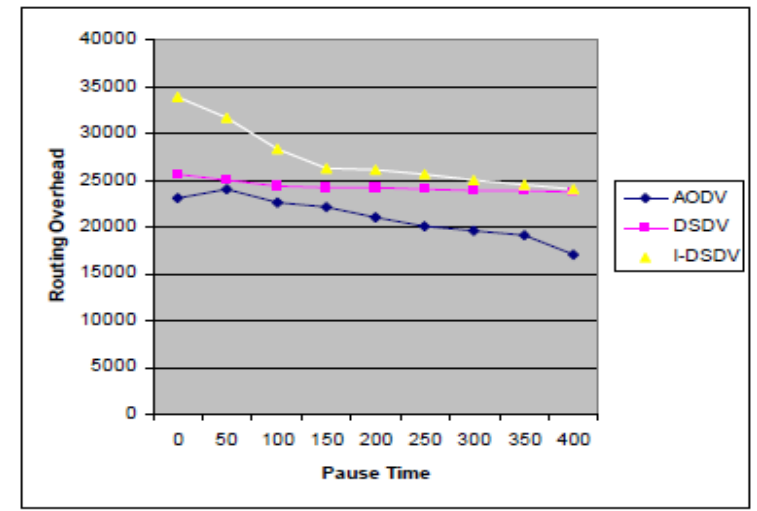

Figure 9: Routing Overhead in Scenario 2

I-DSDV exhibits a flexible property on routing overhead. It needs more routing packets than DSDV all the time, and the difference become smaller as pause time increased. The constant behaviour of DSDV arises as it is a largely periodic routing protocol where routes are mainly established and reconstructed in periodic updates. I-DSDV reduce the frequency of routing update and number of packet generated due to new route establishment when link breaks. AODV generates higher overhead as it used network-wide flooding for route discovery.

\subsection{Various Node Speed}

In this scenario, all the three routing protocol are evaluated based on the three performance metric which are Packet Delivery Fraction, End-to-End Delay and the Routing Overhead. The simulation environments for this scenario are:-

- Various nodes speed which are 10, 20, 30, 40 and $50 \mathrm{~m} / \mathrm{s}$

- Simulation time $=400 \mathrm{~s}$

- Transmission range $=250 \mathrm{~m}$

- Number of nodes is fixed to 30 nodes

- CBR Packet size is set to 1400 Bytes

- Area size is set to $1000 \times 1000$ flat areas

- Random Way Point mobility model is used

\subsubsection{Packet Delivery Fraction}

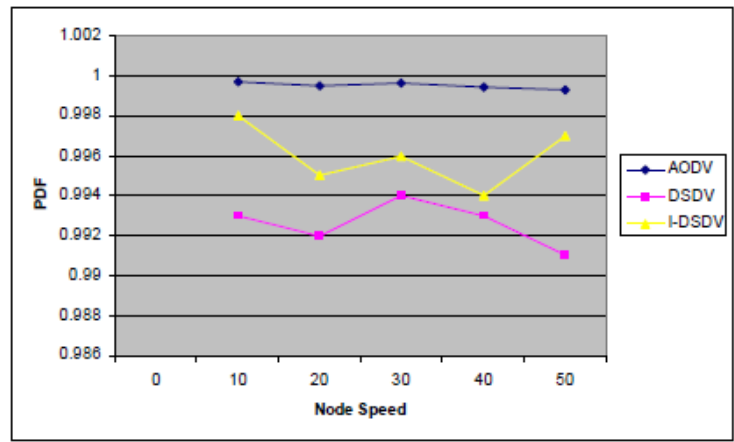

Figure 10: Packet Delivery Fraction in Scenario 3
Based on figure 10, it is shown that the speed of the node has less impact on AODV protocol. IDSDV produces more sent packet as it recover from dropped packet due to link breakage in a higher node speed. DSDV perform less number of packet delivered compared to the other two protocols.

\subsubsection{End-to-End Delay}

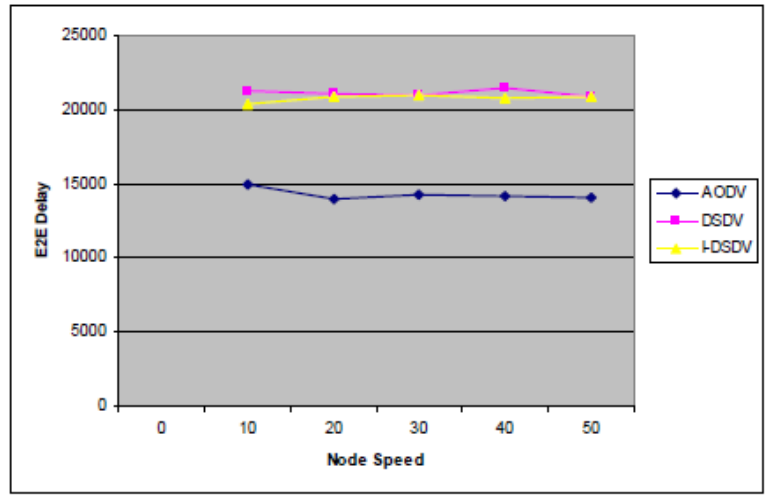

Figure 11: End to End Delay in Scenario 3

Based on Figure above, for varying speed, AODV produces less End to End Delay, but the performance of IDSDV is slightly better than DSDV.

\subsubsection{Routing Overhead}

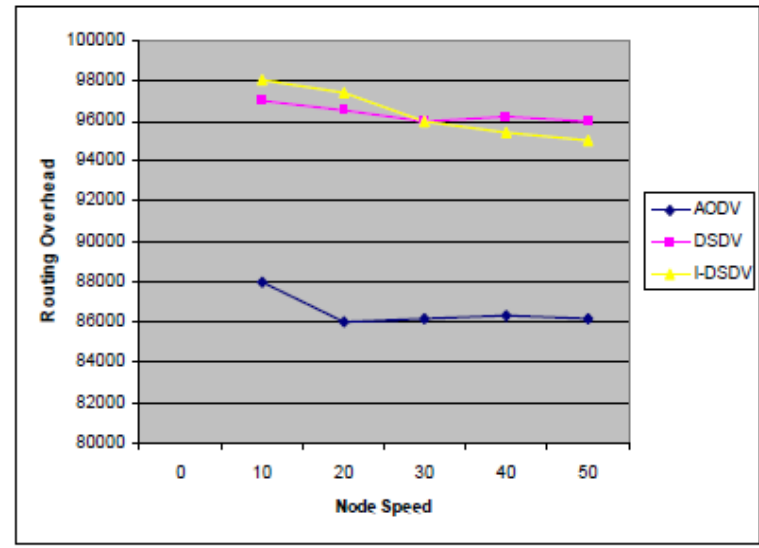

Figure 12: Routing Overhead in Scenario 3

The performance of AODV is far superior compared to the other two. Regular DSDV and IDSDV are almost close to each other for varying number of speed. But I-DSDV is slightly better the regular DSDV when the number is high.

\section{CONCLUSION AND FUTURE WORK}

The performance of all the routing protocol were measured with respect to metrics like Packet Delivery Fraction, End to End Delay and Routing Overhead in three different scenarios: pause time, no of node and node speed. The results indicate that the performance of IDSDV is superior to regular DSDV. It is also observed 
Vol. 5, Issue 12, December 2016

that the performance is better especially when the number of nodes in the network is higher. When the number of nodes increased beyond 30 and above, the performance of both proactive (I-DSDV n DSDV) degenerated due to the facts that a lot of control packets are generated. It is also observed that I-DSDV is even better than AODV protocol in PDF but lower than AODV in E2E delay and Routing Overhead. The reason for the performance to get drop at 20 to 30 nodes is due to varying source and destination nodes and placement barrier in network topology. Simulation also revealed that I-DSDV consume more computation overhead rather than DSDV in the presence of mobility, yielding inferior performance when compared to AODV. It is concludes that I-DSDV improved the PDF and E2E delay when the node is high (link breakage occurred) but still perform lower performance compared to AODV. Since the I-DSDV improved the performance of DSDV, further studies should enhance current I-DSDV in order to achieve higher result. Furthermore, performance comparison with other routing protocol in different classes could be done.

\section{REFERENCES}

[1] C.E. Perkins and P. Bhagwat, "Highly dynamic destinationsequenced distance vector routing (DSDV) for mobile computers," in Proc. ACM SIGCOMM 94, London, UK, Oct. 1994, pp. 234244.

[2] T. Liu \& K. Liu, Improvement on DSDV in Mobile Ad Hoc Networks, IEEE, China, 2007, pp. 1637-1640

[3] J. Broch, D. A. Maltz, D. B. Johnson, Y-Ch Hu, and J. Jetcheva, “A Performance Comparison of Multi-Hop Wireless Ad Hoc Network for mobile ad hoc networks," in Proc. 4th annual IEEE/ACM international conference on Mobile Computing and Networking, Dallas, Texas, U.S.Oct. 1998, pp. 85-97.

[4] C.E. Perkins, E.M. Royer \& S. Das, Ad Hoc On Demand Distance Vector (AODV) Routing, IETFInternet draft, draft-ietf-manet-aodv08.txt, March 2001

[5] C. S. R. Murthy and B. S. Manoj, Ad Hoc Wireless Networks: Architecture and Protocols, ch. Routing Protocols for Ad Hoc Wireless Networks, pp. 299-364. Prentice Hall Communications Engineering and Emerging Technologies Series, New Jersey: PrenticeHall Professional Technical Reference, 2004.

[6] Azizol Abdullah, Norlida Ramly, Abdullah Muhammed, Mohd Noor Derahman: Performance Comparison Study of Routing Protocols for Mobile Grid Environment, pp 82-88, IJCSNS International Journal of Computer Science and Network Security, VOL. 8 No.2, February 2008

[7] Altman E, and Jimenez T., (2003). NS Simulator for Beginners. Lecture notes. Univ.de Los Andes, Merida, Venezuela and ESSI.Sophia-Antipolis, France.

[8] C.E. Perkins \& E.M. Royer, Ad-hoc On-Demand Distance Vector Routing, Proceedings of the 2nd IEEE Workshop on Mobile Computing Systems and Applications, New Orleans, LA, February 1999, pp. $90-100$

[9] K.U.R Khan, A.V. Reddy, R.U. Zaman, K.A Reddy, T.S Harsha, An Efficient DSDV Routing Protocol for WirelessMobile Ad Hoc Networks and its Performance Comparison, Second UKSIM European Symposium on Computer Modeling and Simulation, India, 2008, pp. 506 - 511

[10] A. Boukerche, Performance Evaluation of Routing Protocols for Ad Hoc Wireless Networks, Mobile Networks and Applications 9, Netherlands, 2004, pp. 333-342

[11] A.E. Mahmoud, R. Khalaf \& A, Kayssi, Performance Comparison of the AODV and DSDV Routing Protocols in Mobile Ad-Hoc Networks, Lebanon, 2007

[12] The NS Manual (Oct. 2, 2006), http://www.isi.edu/nsnam/ns.
[13] The Network Simulator NS-2 tutorial homepage, http://www.isi.edu/nsnam/ns/tutorial/index.html

[14] http://en.wikipedia.org/wiki/DestinationSequenced__ Distance_ Vector_routing 